\title{
Precision, double XTEM sample preparation of site specific Si nanowires
}

\author{
L. M. Gignac, S. Mittal, S. Bangsaruntip, G. M. Cohen, and J. W. Sleight
}

IBM T. J. Watson Research Center, 1101 Kitchawan Rd., Yorktown Heights, NY 10598

As semiconductor processing continues to advance, transistors with smaller dimensions and higher packing densities continue to be developed. However, traditional processing methods are approaching their physical limits and advanced device development requires new processing schemes. One method being pursued is fabricating devices from Si nanowires (NW). These devices can be produced from NW with diameters less than $30 \mathrm{~nm}$. To characterize device performance, the devices are electrically tested but the electrical data is often hard to interpret without physical characterization of the specific device that was probed. At these dimensions, the physical characterization is strongly dependent on transmission electron microscope (TEM) imaging. However, TEM sample preparation of a site specific, electrically tested Si NW is a challenge that requires state-of-the-art dualbeam focused ion beam (DB-FIB) equipment and a skilled operator.

In this paper, a TEM sample preparation method will be discussed where a site specific, electrically tested, sub-30 nm diameter Si NW device structure was sectioned first parallel and then perpendicular to the NW direction. The Si NW device, shown in the scanning electron microscope (SEM) image in Fig. 1a, was produced using electron-beam (e-beam) lithography and standard semiconductor processing methods. Coincident measurements of the wire cross-section, gate dielectric thickness, and gate length needed to be taken from the same NW device but these measurements required TEM cross-sections (XTEM) $90^{\circ}$ from one another. To be able to measure these parameters, an XTEM sample was initially produced in the direction along the NW length, see the TEM image in Fig. 1b. This sample was prepared using ex-situ FIB and lift-out onto a C support membrane [1]. From this sample, the gate length was determined to be $70 \mathrm{~nm}$. After TEM imaging of this sample was complete, it was put back in the DB-FIB and sectioned a second time perpendicular to the NW direction. The TEM grid with the first XTEM sample sitting on a $\mathrm{C}$ membrane was mounted on the DB-FIB flip stage with careful attention being paid to the sample orientation with the grid lying flat, $90^{\circ}$ from the e-beam direction. After e-beam Pt was deposited on the sample surface, an in-situ probe was attached to the XTEM sample and the C membrane was cut away from the edges of the sample. The sample was then attached to the side finger of an Omniprobe $\mathrm{Cu}$ grid and the sample was sectioned in the FIB until the NW was seen in the SEM on both sides of the sample, see Fig. 2a-b. TEM images of the second XTEM sample is shown in Fig. 3a-b. The Si NW cross-sectional dimensions were measured to be $28.5 \mathrm{~nm}$ laterally and $21.5 \mathrm{~nm}$ vertically. The FIB damage of Si which was generated from the first XTEM sample preparation is shown in Fig. 3a. The high resolution TEM image in Fig. 3b demonstrates that the number of $\mathrm{Si}$ atom columns forming the NW device can be counted and conduction plane modeling can be attempted. Double sectioning TEM sample preparation has been demonstrated previously $[2,3]$ but not to section specific, sub-30 $\mathrm{nm}$ features in both directions. Electron tomography could also be used for similar 3-dimensional characterization of Si NW devices.

\section{References}

[1] L.A. Giannuzzi, J.L. Drown, S.R. Brown, R.B. Irwin, F.A. Stevie, Proc MRS, 480, 1997, 19-27.

[2] J.C. Lee, D. Su, and J.H. Chuang, Microelectronics Reliability, 41, 2001, 1551-1556.

[3] N. Wang and S. Li, Electronic Device Failure Analysis, 10, 2008, 12-16. 

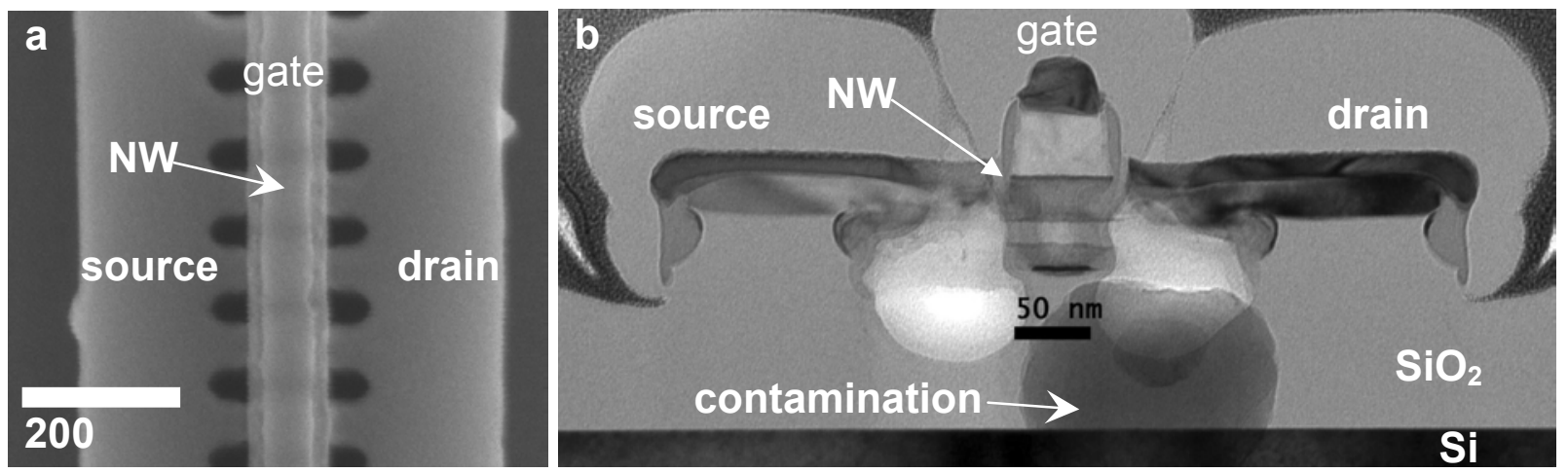

Fig. 1: a) top down SEM image of Si NW device and b) TEM image of Si NW crosssectioned parallel to a sub-30 nm diameter Si NW device with a $70 \mathrm{~nm}$ gate length.

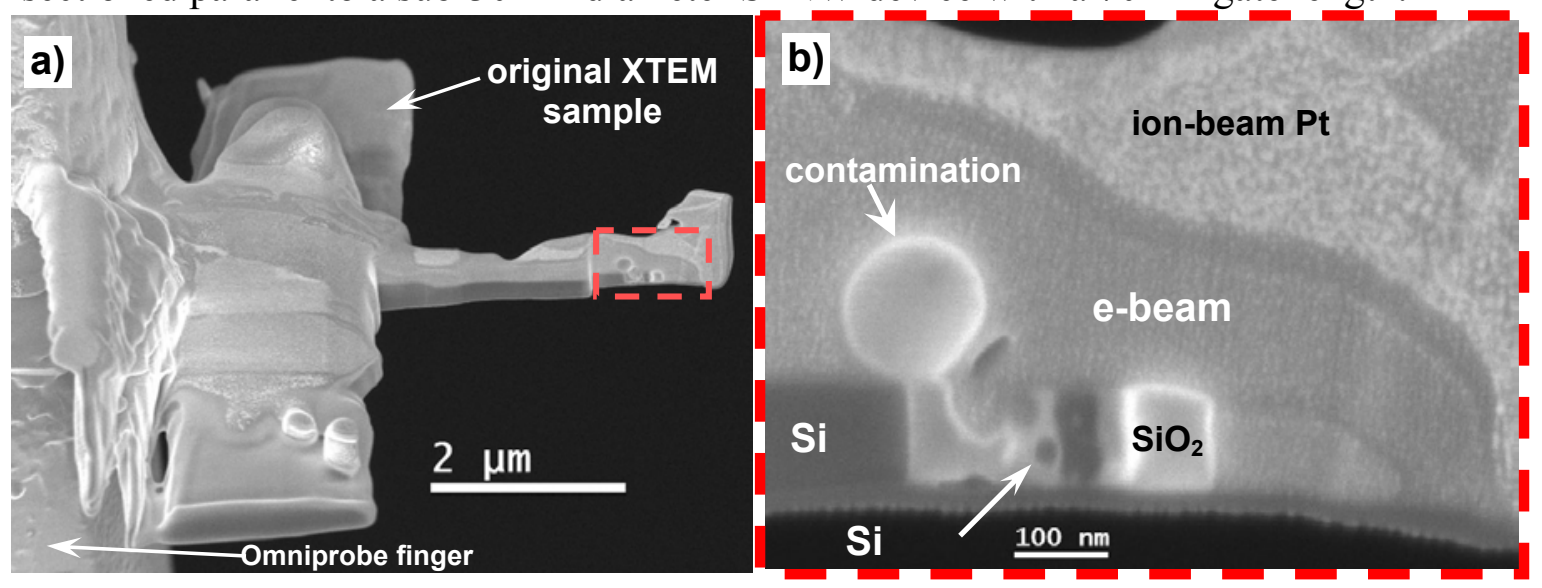

Fig. 2: a) SEM image of a XTEM sample sectioned perpendicular to the NW direction prepared from an XTEM sample sectioned parallel to the NW direction (shown in Fig. 1b); b) higher magnification SEM image of the final XTEM sample showing the Si NW.

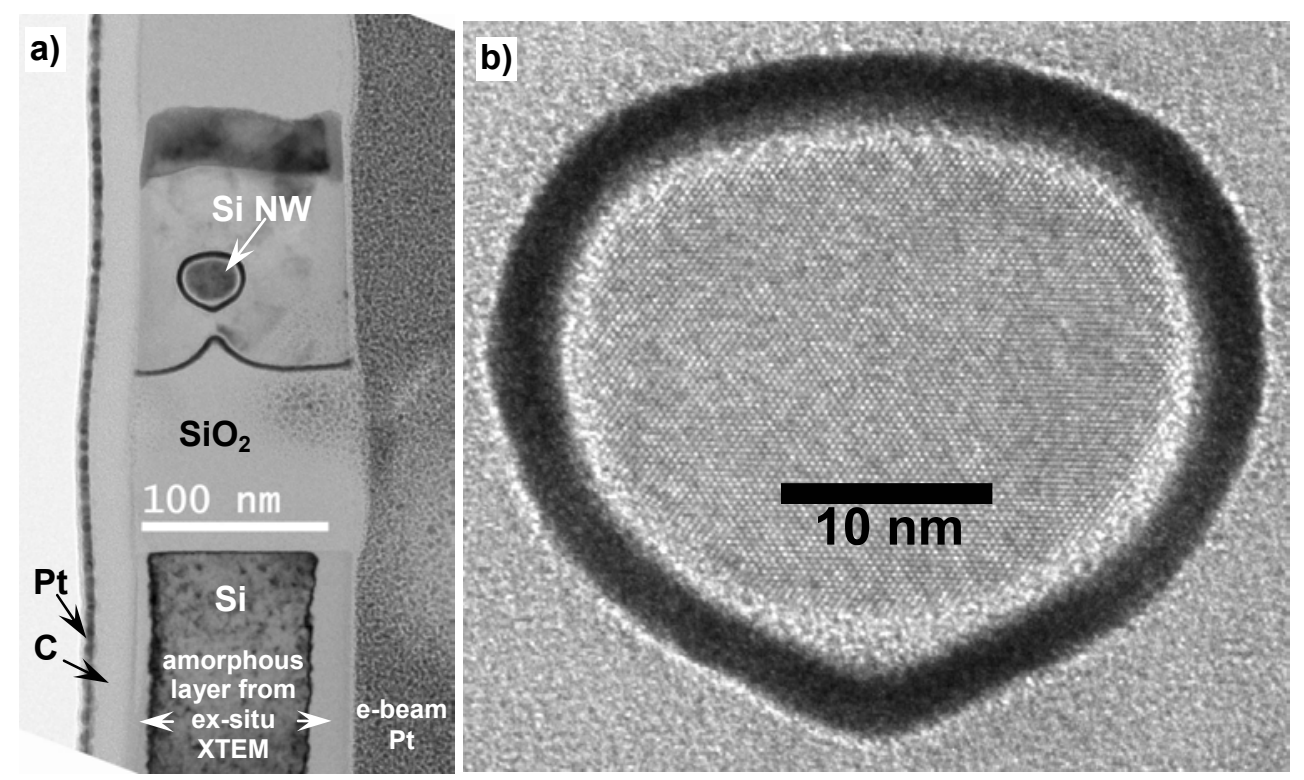

Fig. 3: a) TEM image of the XTEM sample prepared from an XTEM sample. The Si NW is $28.5 \mathrm{~nm}$ wide and $21.5 \mathrm{~nm}$ high. b) high resolution TEM image of the Si NW. 